\title{
Téoros
}

Revue de recherche en tourisme

\section{Destination Montréal : d'hier à demain}

\section{Pierre Bellerose}

Volume 10, numéro 3, novembre 1991

Destination Montréal : d’hier à demain

URI : https://id.erudit.org/iderudit/1079193ar

DOI : https://doi.org/10.7202/1079193ar

Aller au sommaire du numéro

Éditeur(s)

Université du Québec à Montréal

ISSN

0712-8657 (imprimé)

1923-2705 (numérique)

Découvrir la revue

Citer ce document

Bellerose, P. (1991). Destination Montréal : d'hier à demain. Téoros, 10(3), 2-2. https://doi.org/10.7202/1079193ar d'utilisation que vous pouvez consulter en ligne.

https://apropos.erudit.org/fr/usagers/politique-dutilisation/ 


\section{Présentation}

\section{Destination Montréal : d'hier à demain}

\begin{abstract}
"Montreal is one of those cities that inspire in their aficionados the kind of sentiment one feels for the other partner inaloveaffair that has beencharming and perhaps difficult, but is not yet completely finished and over. Faded grandeur and remembrance of things past, true; but you keep thinking also that there's more to come. And with Montréal, there always is."
\end{abstract}

Margaret Atwood, woie de vivre of Montréals, New York Time Magazine, March 13, 1983.

Réaliser un numéro spécial sur le tourisme a Montréal est un projet fort ambitieux. En effet, Montréal a été une destination trés étudiée comme en fait foi un recensement fait par le Centre d'études du tourisme en 1987 qui dénombrait près de 300 études rendues publiques depuis 1980 sur une des différentes facettes de la problématique touristique montréalaise. La plupart des constats faisaientressortirlevieillissement important du produit touristique, mettaient aussi l'emphase sur les problèmes majeurs de leadership et de concertation entre les differents intervenants touristiques de la région de Montréal et finalement, insistaient sur le sous-financement chronique de la promotion touristique.

La revue Téoros ne pouvait choisir un meilleur moment, a laube du 350e anniversaire de Montreal, pour consacrer un numéro à la deuxième plus importante ville francophone au monde. En effet, depuis environ deux (2) ou trois (3) ans, plusieurs changements importants sont survenus, permettant ainside modifier le portrait de la réalité touristique mon tréalaise. La démarche stratégique sur le tourisme a Montreal, entreprise en 1986, a amenéla fusion en 1989 de la CIDEM-Tourisme Ville de Montréall a l'Office des congres et du tourisme du Grand Montreal (OCTGM). De plus, l'intégration, au début de l'année 1992, de la Société d'accueilet de renseignements touristiques (SARTM) à I'OCTGM viendra terminer le travail accompli précédemment et ainsi consolider toutes les forces vives du tourisme au sein du même organisme.

Cette consolidation autour de l'OCTGM permet enfin ả cet organisme d'assumer son rôle de leader et de travailler efficacement à la concertation.

Pour illustrer les changements au niveau du produit touristique, j'aborde dans le premier article de ce numéro l'évolution du produit montréalais en faisant ressortir les differrents projets en cours de realisation et ceux qui seraient souhaitables dans un avenir plus ou moins rapproché. Par la suite, Marcel Le Sieur analyse le processus de transfert des aéroports de Montreal vers une autorité locale qui ne pourra qu'aider, encore là, à assumer un plus grand leadership.

De son côté, Sandrine Brilletétablitune relation trés intéressante entre le réseau piétonnier intérieur et le produit touristique du centre-ville. Elle arrive ainsi à nous démontrer l'importance de ce réseau pour notre industrie. L'article de Jean Stafford sur I'hôtellerie a Montréal illustre bien l'évolution qu'a connue ce secteur depuis quelques années et la problématique que vit actuellement l'hôtellerie prise entre une offre renouvelée et une demande stagnante.

Les articles de Marie-Andrée Camirand et François Cadoret analysentdeux secteurs fondamentaux et souventsous-estimés du tourisme montréalais soit les congres et les foires commerciales. Les deux (2) auteurs en arrivent à la même conclusion pour leurs secteurs respectifs: nous avons besoin de nouvelles infrastructures.

Pendant que Jean-Marc Bissonnette présente le projet de signalisation touristique, tant piétonnière que routière, de la Ville de Montréal, Chantal Langelier étudie les tendances de la clientèle d"agrément à Montréal. Son article démontre que le déclin apparent de la clientèle d'agrément cache peut-être des changements d'habitudes de voyages des nord-américains.

Pierre Labrie est le premier à estimer les effets à court et long termes des célébrations du 350 e anniversaire de Montréal sur le tourismeendemontrantqueles retombées surnotre industrie seront peut-être plus importantes que l'on croit. Par la suite, je présente le projet, actuellement en cours, d'un système d'indicateurs de performance du tourisme à Montréal, qui permettra enfin de mieux cerner l'activité touris tique de la ville. Guy Morin livre sa vision du nouveau Póle Maisonneuve ainsi que les défis qui attendent cette zone touristique. Pour compléter ce numéro, un article de Louis St-Jean surl'image et la perception de Montréal fait ressortir les differences de perception qui existent selon les clientèles.

En définitive, malgré la récession actuelle, les problèmes du Stade olympique et les ratés des premiers pas des célébrations du 350 e anniversaire de Montreal, plusieurs facteurs nous indiquent que nous avons parcouru beaucoup de chemin depuis le milieu des annees 1980 . Le produit touristique montréalais est en voie de se renouveler de façon importante. De plus, I'OCTGM a atteint un niveau de leadership inégalé depuis trente $(30)$ ans, ce quia permis d"améliorer grandement le climat de concertation. Malheureusement, un élémentreste encore sans solution: le sous-financement de la promotion touristique. II s'agit du problême fondamental qui empêchera peut-être Montréal, au sortir de la récessionactuelle, d'accroitre de façon substantielle sa clientèle et sa part de marché. Le financement de la promotion demeure le point central sur lequel doivent se concentrer les differrents intervenants, tant privés que publics, si l'on veut que l'industrie touristique montréalaise atteigne un niveau de croissance al la mesure de son potentiel. 